\title{
AGRESSIVIDADE, ESTILO DE VIDA CRIMINAL E ADAPTAÇÃO À PRISÃO
}

\author{
Leonel Cunha Gonçalves e Rui Abrunhosa Gonçalves
}

Resumo: O estudo da adaptação à prisão e a classificação dos reclusos pode ajudar a prevenir situações de risco e promover um tratamento penitenciário mais efetivo. Este estudo correlacional explora a relação entre a agressividade, o estilo de vida criminal e a adaptação à prisão, incluindo variáveis sociodemográficas e jurídico-penais. A agressividade é medida pelo Aggression Questionnaire e o estilo de vida criminal pelo Lifestyle Criminality Screening Form. A adaptação à prisão inclui dois indicadores: processos disciplinares e acessos aos serviços clínicos. A amostra é constituída por trinta e um participantes do sexo masculino e nacionalidade portuguesa, detidos num Estabelecimento Prisional Regional. O período de observação teve a duração de cinco meses. Os resultados indicam que a agressividade, a história de consumo de drogas e as penas de menor duração predizem pior adaptação à prisão. Conclui-se que o Aggression Questionnaire se associa aos processos disciplinares e aos acessos aos serviços clínicos.

Palavras-chave: Adaptação. Agressividade. Estilo de vida. Prisão.

\section{Introdução}

Existe grande interesse em compreender como é que os reclusos se adaptam à vida na prisão (Dhami, Ayton, \& Loewenstein, 2007), pois a reclusão é a forma convencional de lidar com os ofensores mais perigosos (Zamble \& Porporino, 1990) e as taxas de encarceramento têm vindo a crescer em diversas partes do mundo (Walmsley, 2009). Ao longo dos tempos, fenômenos tais como a superlotação, a 
violência, as drogas, a ineficácia da prisão na readaptação dos reclusos e o descontentamento tanto dos funcionários prisionais como da opinião pública alertaram para a necessidade da utilização de modelos de classificação prisional que enfatizem questões de segurança, planejamento financeiro e otimização dos recursos (MacKenzie, 1989). A utilização de instrumentos psicométricos e a classificação dos reclusos pode promover a segurança, determinando regimes de cumprimento da pena apropriados ao nível de periculosidade dos sujeitos e pode também promover a reabilitação, sinalizando programas prisionais apropriados às necessidades criminógenas de cada um, num estádio precoce da sua detenção (e.g. Motiuk, 1997).

Embora varie de indivíduo para indivíduo e tenha caráter reversível, a adaptação à prisão é um processo complexo ao nível psicológico e pode mesmo causar perturbações de stress pós-traumático após a libertação (Haney, 2003). Como "instituições totais" (ver Goffman, 1961) as prisões sujeitam o comportamento humano ao controle espacial e temporal, através de hierarquias de vigilância, das regras penitenciárias e da rotina prisional (Coleman, 2008). Ao mesmo tempo, os reclusos têm de aceitar as normas e os valores da população prisional como estratégia de sobrevivência, através da qual aprendem a utilizar o sistema e a subcultura carcerária em proveito pessoal (Jewkes, 2007). Consequentemente, a reclusão é uma experiência de vida traumática que requer modificações nos padrões básicos de vida e de funcionamento dos indivíduos (Harding \& Zimmerman, 1989).

Desde a teoria da "Prisionização" de Clemmer (1940) até aos dias de hoje, foram escritos inúmeros livros e artigos sobre a vida nas prisões (e.g. Jiang \& Fisher-Giorlando, 2002). Ao nível teórico, os diferentes perfis de adaptação à prisão dos reclusos têm sido explicados em função das vivências pessoais e dos valores culturais que estes trazem do exterior (Teoria da "Importação" - Irwin \& Cressey, 1962; Schrag, 1961) ou pela pressão vivida na prisão e características específicas dos diferentes estabelecimentos prisionais (Teoria da "Privação" - Goffman, 1961; Sykes, 1958). Na década de 1980, graças aos avanços estatísticos e informáticos, os investigadores passaram a procurar sistematicamente preditores da adaptação à prisão dos reclusos, considerando, entre outros fatores, a agressividade e o estilo de vida criminal (e.g. Carbonell, Megargee, \& Moorhead, 1984; Lahm, 2008; Walters, 2005, 2007).

O comportamento agressivo é frequentemente associado às infrações institucionais, embora possa ser utilizado como uma estratégia de adaptação à prisão, onde os estressores são comuns e o ambiente reduz a escolha de estratégias disponíveis para lidar com os problemas (Ireland, Brown, \& Ballarini, 2006). Num estudo com reclusos detidos na Espanha, Rodríguez-Fornells, Lopez-Capdevila e Andres-Pueyo (2002) verificaram que a agressividade, medida pelo Aggression Questionnaire (AQ - Buss \& 
Perry, 1992), apresenta uma correlação positiva significativa com a ocorrência de processos disciplinares muito graves, mesmo após controlarem o efeito da desejabilidade social no instrumento. Também Lahm (2008), utilizando alguns itens do mesmo questionário, comprovou uma relação positiva significativa com a ocorrência de confrontos físicos entre reclusos. Assim, supomos que os reclusos que relatem níveis mais elevados de agressividade se associem a uma pior adaptação à prisão.

Presume-se ainda que os indivíduos com maior estilo de vida criminal possam associar-se a uma adaptação negativa à prisão. Devido a sua reiterada associação com grupos e atividades delinquentes, alguns ofensores possuem fortes atitudes e identidades criminais, sendo assim mais suscetíveis ao envolvimento em comportamentos disruptivos (Hochstetler \& DeLisi, 2005). Em alguns dos seus estudos sobre a adaptação à prisão, Walters $(2005,2007)$ verificou que o estilo de vida criminal, medido pelo Lifestyle Criminality Screening Form-Revised (LCSF-R - Walters, 1995), se correlaciona com os processos disciplinares graves (envolvendo violência), mas não com os processos disciplinares simples (não envolvendo violência), nem com o número total das infrações cometidas.

De acordo com estudos realizados em Portugal (Gonçalves, 1993, 2008), existem quatro diferentes tipos de reclusos quanto a sua adaptação prisional: os reclusos "bem-adaptados", os "mal-adaptados", os "sobre-adaptados" e os "inadaptados". Os reclusos bem-adaptados não possuem geralmente antecedentes criminais, estão detidos por crimes ocasionais embora violentos, têm idade superior aos trinta anos, apresentam valores convencionais e não se envolvem em processos disciplinares. Por sua vez, os mal-adaptados são frequentemente reincidentes, têm idade média de vinte e nove anos, manifestam comportamentos agressivos, violação das regras institucionais e ostentam valores antissociais. Os reclusos sobre-adaptados têm maior cadastro criminal, possuem passado institucional, têm uma média de idade acima dos trinta e cinco anos, demonstram um comportamento assertivo na prisão mas podem pertencer a grupos delinquentes, procuram a maximização de benefícios e são de difícil reinserção social. Por fim, os reclusos inadaptados manifestam um desajustamento global à prisão através de patologias de adaptação, stress prisional, comportamento autodestrutivo, consumo de drogas, isolamento social, ataques de cólera, têm uma média de idade de vinte e quatro anos e são de fácil vitimização.

As críticas às classificações tipológicas são sobejamente conhecidas, nomeadamente por terem um caráter estático e dificilmente ser possível encontrar tipos "puros" (e.g. Garrido, 1993; Gonçalves, 2008), o que, no estudo presente, poderá contribuir para a existência de reclusos tipificados como "borderline", ou seja, sujeitos com dificuldade de controle dos impulsos, baixa capacidade de tolerância à frustração, perturbação da identidade, labilidade emocional e humor disfórico (American Psychiatric 
Association, 2002). Estes sujeitos demonstram uma forma de adaptação em que as tipologias de "mal-adaptados" e"inadaptados" se sobrepõem.

Para além disso, verifica-se desconfiança quanto à utilização de instrumentos de autorrelato na literatura prisional.Devido aos fenômenos de desejabilidade social, muitos investigadores e técnicos preferem utilizar instrumentos em que os relatos do recluso sejam minimizados. Porém, estudos recentes têm comprovado a validade dos questionários na investigação com ofensores (e.g. Gallardo-Pujol et al., 2006; Walters, 2005, 2007).

No presente trabalho defendemos a ideia de que os instrumentos de avaliação e as tipologias de classificação possibilitam resultados essenciais tanto para a avaliação dos reclusos como para o tratamento penitenciário. Neste sentido, a sistematização de conhecimentos poderá contribuir para a elaboração de uma metodologia de avaliação estandardizada e cientificamente sustentada que ajude os técnicos a decidir acerca do nível de periculosidade do recluso, indicar planos efetivos de tratamento penitenciário, regular os pareceres sobre medidas flexibilizadoras da pena e planejar a sua progressiva reinserção na comunidade.

\section{Objetivos}

Dadas as dimensões da amostra, este estudo tem um caráter exploratório mas que ainda assim pretende: 1) Identificar preditores da adaptação à prisão dos reclusos; 2 ) Identificar variáveis sociodemográficas e jurídico-penais relacionadas com a agressividade e o estilo de vida criminal; 3) Comparar a eficácia de diferentes tipos de instrumentos (autorrelato vs. atuarial) na predição da adaptação à prisão; 4) Caracterizar diferentes tipologias de adaptação; e 5) Indicar recomendações para o tratamento e gestão penitenciária dos reclusos.

\section{Metodologia}

\section{Instrumentos e variáveis}

Este estudo recorre a dois instrumentos psicométricos: o Aggression Questionnaire (Buss \& Perry, 1992) e o Lifestyle Criminality Screening Form Revised (Walters, 1998). O AQ é um instrumento de autorrelato que mede a agressividade. Possui escala tipo likert podendo pontuar de 1 até $5 \mathrm{em}$ cada um dos seus 29 itens. As dimensões deste instrumento surgiram de análise fatorial que identificou 4 fatores: a agressividade verbal e a 
agressividade física, a raiva e a hostilidade. Estes fatores parecem estar inter-relacionados, variando na intensidade, frequência e duração (GarcíaLeón et al., 2002) e, segundo a teoria subjacente a este instrumento, esta divisão categorial estende-se aos traços de personalidade. Não existem pontos de corte, pelo que quanto mais o indivíduo pontua maior é o nível de agressividade que possui.

O LCSF-R é um instrumento de avaliação do tipo atuarial e em formato de checklist. Mede o estilo de vida criminal e tem sido utilizado no estudo da adaptação à prisão e da reincidência no crime (e.g. Walters, 2005 , 2007). Este instrumento é cotado mediante a informação disponível nos ficheiros institucionais dos reclusos. Contém 14 itens, podendo o sujeito pontuar 0, 1 ou 2 em cada um deles. Uma pontuação total superior a 10 é, segundo Walters (1998), reveladora da existência de um estilo de vida criminal. Segundo o racional teórico exposto pelo autor, o estilo de vida criminal manifesta-se através da conjunção de 4 modos de expressão comportamental, que correspondem a outros tantos fatores: a irresponsabilidade, a autoindulgência, o comportamento interpessoal intrusivo e a violação de regras sociais.

A adaptação destes instrumentos para a população portuguesa revelou-se globalmente ajustada no caso do AQ (e.g. Simões, 1993), existindo alguns estudos com o LCSF-R que atestam a sua utilidade em amostras forenses portuguesas (e.g. Gonçalves \& Lopes, 2007; Gonçalves \& Vieira, 2005; Vieira, Lopes, \& Gonçalves, 2008). Além disso, são instrumentos relativamente breves e de rápida cotação, o que pode ajudar os técnicos e diretores dos estabelecimentos prisionais a tomar decisões ajustadas no espaço de tempo limitado da avaliação inicial dos reclusos.

Com a finalidade de se avaliar a adaptação à prisão dos reclusos consideramos duas variáveis dependentes, julgadas demonstrativas deste construto: o número de processos disciplinares e o número de acessos aos serviços clínicos. Os processos disciplinares são a medida mais usada no estudo da adaptação à prisão (Wright, 1985). Um maior número de processos disciplinares é sinónimo de uma pior adaptação. Neste estudo, foram considerados todos os processos disciplinares em que os sujeitos foram sancionados (durante o período de observação), independentemente do tipo de sanção e da natureza da ocorrência. Por sua vez, o número de acessos aos serviços clínicos é menos utilizado no estudo da adaptação à prisão mas não deixa de ser importante, uma vez que as queixas por doença/ferimentos estão relacionadas com stress e ansiedade e fornecem por isso uma medida mais sintomatológica do processo de adaptação (Wright, 1985). Assim, consideramos que os reclusos que acedem mais vezes aos serviços clínicos têm maiores dificuldades de adaptação. Estão englobados nesta variável todos os acessos ao médico e enfermeiro dentro do estabelecimento prisional, bem como as consul- 
tas/tratamentos em instituições exteriores, incluindo as consultas de psiquiatria/psicologia.

Para além de analisarmos individualmente estes dois indicadores da adaptação à prisão, construímos uma grelha que os engloba numa medida geral. Para tal, calculou-se a média total da amostra em cada indicador e delimitaram-se os pontos de corte com base em intervalos de desvio padrão. Os resultados totais pontuam assim entre 2 e -4 , sendo que resultados iguais ou superiores a 0 identificam os reclusos adaptados, enquanto os resultados negativos identificam os reclusos não adaptados. Os reclusos com pontuações negativas ao nível dos processos disciplinares representam os reclusos mais agressivos ou "mal-adaptados", enquanto reclusos com pontuações negativas nos acessos aos serviços clínicos configuram os reclusos mais apelativos ou "inadaptados", segundo a tipologia de Gonçalves $(1993,2008)$. Reclusos com pontuações negativas mistas serão considerados "borderline".

Para além das variáveis já mencionadas, este estudo analisa ainda outras de natureza sociodemográfica (idade, estado civil, habilitações literárias e história de consumos) e jurídico-penal (situação penal, tipo de crime, antecedentes criminais, tempo de condenação, medidas flexibilizadoras e ocupação dentro do estabelecimento prisional).

\section{Participantes}

Este estudo é realizado a partir de uma amostra de conveniência composta por 31 reclusos do sexo masculino de nacionalidade portuguesa, detidos num Estabelecimento Prisional Regional do norte de Portugal, que foram observados por um período de cinco meses.

Ao nível das variáveis sociodemográficas, a idade média da amostra situa-se nos 37 anos, tendo o sujeito mais novo 19 anos e o mais velho $49(d p=7.92)$. As faixas etárias mais representadas são as dos 31-40 anos e dos 41-50, com 12 reclusos cada (38.7\%), constando ainda 7 reclusos com menos de 30 anos (22.6\%). Relativamente à escolaridade, 16 sujeitos possuem o $6^{\circ}$ ano (51.6\%), 9 possuem a $4^{\text {a }}$ classe (29\%), 3 têm menos do que a escolaridade primária completa (9.7\%) e outros 3 possuem o $9^{\circ}$ ano (9.7\%). Quanto ao estado civil, 13 dos sujeitos são solteiros (41.9\%), 12 são casados (38.7\%) e 6 são divorciados (19.4\%). Verifica-se ainda que 14 reclusos (45.2\%) não apresentam história de consumos, 6 (19.4\%) têm história de consumo de álcool, 3 (9.7\%) têm história de consumo de drogas e $8(25.8 \%)$ apresentam história de consumos mistos (álcool e drogas).

No domínio jurídico-penal, 10 dos reclusos são preventivos (32.3\%) e 21 (67.7\%) são condenados. Quanto aos antecedentes criminais, 14 são primários (45.2\%) e 17 são reincidentes (54.8\%). No que concerne ao tipo de crime, 11 sujeitos estão atualmente condenados por crimes contra as pessoas (35.5\%), 10 cometeram crimes contra o património (32.3\%), 6 
praticaram crimes relativos a estupefacientes (19.4\%) e 4 foram condenados por outros tipos de crime (12.9\%). No momento de avaliação, 21 dos sujeitos não usufruíam de medidas flexibilizadoras (74.2\%), 3 se beneficiavam de saídas precárias (9.7\%), outros 3 têm Regime Aberto Interior (9.7\%) e 2 estão em Regime Aberto Exterior (6.5\%). A média de tempo de pena da amostra é de 43.43 meses de prisão, sendo a pena mínima 8 meses e a máxima $78(d p=22.88)$.Verifica-se que 16 reclusos $(51.6 \%)$ não têm qualquer ocupação dentro da prisão, 12 (38.7\%) trabalham, 2 (6.5\%) frequentam a escola e apenas 1 (3.2\%) trabalha e frequenta a escola simultaneamente. No que diz respeito às visitas, num espaço de cinco meses, a média é 35.52 (7 visitas por mês, $d p=33.94$ ).

\section{Procedimento}

Os reclusos selecionados foram individualmente convocados com o objetivo de se lhes expor os propósitos do estudo, assegurar os princípios de confidencialidade dos dados e requisitar a sua colaboração. Após assinarem o consentimento informado, os voluntários foram individualmente dirigidos para um gabinete onde preencheram o AQ. Após recolher os dados, cotamos o AQ, o LCSF-R e a grelha de adaptação à prisão. Mais tarde, procedeu-se à recolha dos dados sociodemográficos e jurídico-penais dos reclusos. Neste procedimento, consultamos diversas fontes institucionais: dossiês dos reclusos, registos do Serviço de Vigilância e registos do Serviço de Educação e Ensino.

As análises descritivas e os testes correlacionais foram executados no programa SPSS 17. Posteriormente, devido à necessidade de realizar análises de regressão mais ajustadas às variáveis dependentes deste estudo (dados de contagem com elevada dispersão), recorremos ao software STATA 11. Neste programa realizamos vários tipos de análises de regressão (lineares e não lineares) para compararmos a adequabilidade de diferentes modelos. Em seguida, calcularam-se análises robustas da variância e as mudanças esperadas nas variáveis dependentes em função dos preditores considerados.

\section{Resultados}

Num primeiro momento apresentamos os resultados obtidos pela amostra nos diferentes instrumentos de medida utilizados. Posteriormente, expõem-se os principais resultados estatísticos de acordo com os objetivos referidos. Por último, apresenta-se a grelha de avaliação da adaptação à prisão e a pontuação dos reclusos não adaptados nos dois 
indicadores considerados. Na Tabela 1 são descritos os resultados do AQ, do LCSF-R e da grelha de adaptação à prisão.

Tabela 1

Resultados das medidas de avaliação

\begin{tabular}{lcccc}
\hline & Mínimo & Máximo & Média & Desvio Padrão \\
\hline Agressividade física & 9 & 32 & 17.19 & 6.97 \\
Agressividade verbal & 7 & 19 & 13.26 & 3.13 \\
Raiva & 7 & 28 & 14.29 & 5.09 \\
Hostilidade & 12 & 31 & 21.13 & 5.18 \\
Agressividade (total) & 42 & 90 & 65.87 & 14.65 \\
Irresponsabilidade & 0 & 4 & 1.58 & 1.12 \\
Auto-indulgência & 0 & 5 & 2.06 & 1.57 \\
Comportamento interpessoal intrusivo & 0 & 5 & 1.06 & 1.46 \\
Violação regras sociais & 0 & 3 & 0.84 & 0.74 \\
Estilo vida criminal (total) & 0 & 12 & 5.55 & 2.57 \\
Processos disciplinares & 0 & 10 & 0.77 & 2.08 \\
Acessos aos serviços clínicos & 0 & 19 & 4.03 & 4.63 \\
Adaptação à prisão (total) & 0 & 22 & 4.81 & 5.56 \\
\hline
\end{tabular}

Na escala total da agressividade, a média da amostra é $65.87(d p=$ 14.65), sendo o valor mínimo 42 e o máximo 90 . Ao nível dos fatores do instrumento, a agressividade física apresenta uma média de 17.19 ( $d p=$ 6.97), a agressividade verbal $13.26(d p=3.13)$, a raiva $14.29(d p=5.09)$ e a hostilidade é a escala com média superior: $21.13(d p=5.18)$. Os autores do estudo original de validação do AQ (Buss \& Perry, 1992) obtiveram para o gênero masculino a média de $77.8(d p=16.5)$. No presente estudo, a média obtida pelos reclusos é bastante inferior à do estudo original e à da aferição realizada por Simões (1993), que foi 75.67 .

Quanto ao total do estilo de vida criminal, a média situa-se nos 5.55 $(d p=2.57)$, sendo o valor mínimo 0 e o máximo 12. Na escala de irresponsabilidade a média é $1.58(d p=1.12)$. O tipo de estilo de vida criminal com média superior nesta amostra é a autoindulgência, com o valor de $2.06(d p=1.57)$. Na escala de comportamento interpessoal intrusivo a média é $1.06(d p=1.46)$ e na violação de regras sociais, $0.84(d p=0.74)$. Dos 
31 reclusos desta amostra, apenas 2 revelaram resultados superiores a 10 pontos (6.45\%), indicador do estilo de vida criminal. A aplicação deste instrumento a outra amostra de reclusos portugueses (Vieira, Lopes, \& Gonçalves, 2008) evidenciou uma estrutura fatorial não inteiramente coincidente com a proposta do autor (Walters, 1995). Nesse estudo, a média da amostra na escala total do instrumento foi $5.1(d p=4.40)$ para as mulheres e $6.18(d p=3.05)$ para os homens, o que é semelhante aos resultados aqui apresentados.

Relativamente à adaptação à prisão, a média da amostra no total da grelha de avaliação (soma dos processos disciplinares e acessos aos serviços clínicos) é 4.81 ( $d p=5.56$ ), sendo o valor mínimo 0 e o máximo 22 (valores mais elevados representam pior adaptação). $O$ número de acessos aos serviços clínicos apresenta média de $4.03(\mathrm{dp}=4.63)$ e os processos disciplinares a média de $0.77(d p=2.08)$.

No intuito de responder ao primeiro objetivo do estudo, começamos por realizar regressões lineares a fim de identificar os preditores mais significativos da adaptação à prisão. No que toca aos processos disciplinares, constatamos que os dois preditores que explicam maior variância nesta variável dependente são a história de consumo de drogas $\left(R_{a j}^{2}=0.17, F_{(1,29)}=7.16, p=0.012\right)$ e a agressividade $\left(R_{a j}^{2}=0.10, F_{(1,29)}=4.3\right.$, $p=0.047$ ). Porém, a variável dependente "processos disciplinares" é uma variável de contagem e não cumpre, nesta amostra, os pressupostos para a realização de regressões lineares, alertando-nos para a necessidade de utilizar modelos de regressão não lineares. Assim, comparamos diferentes modelos no intuito de selecionar aquele que melhor se ajustava às nossas variáveis. Na Tabela 2 apresentamos os três diferentes modelos explorados (Ordinary Least Squares, Poisson e Negative Binomial).

Tabela 2

Modelos de regressão para as variáveis Processos disciplinares, História consumo de drogas e Agressividade.

\begin{tabular}{cccc}
\hline Modelo & LL & gl & BIC \\
\hline Ordinary Least Squares & -61.23 & 3 & 132.76 \\
Poisson & -27.94 & 3 & 66.19 \\
Negative Binomial & -24.81 & 4 & 63.35 \\
\hline
\end{tabular}

LL:Log-likelihood

gl: graus de liberdade

BIC: Bayesian Information Criterion 
Como seria de se esperar, por serem modelos concebidos para analisar variáveis dependentes de contagem, os modelos Poisson e $\mathrm{Ne}$ gative Binomial apresentam melhores resultados. Entre estes modelos, 0 Negative Binomial é aquele que melhor se adapta às nossas variáveis (LL e BIC inferiores) pelo que foi escolhido. Este modelo, em que se analisa a história de consumo de drogas e a agressividade ( $A Q$ - total) enquanto preditores do número de infrações institucionais, apresenta um Pseudo $R^{2}$ de 0.20 e é estatisticamente significativo, mesmo após calcularmos estimativas robustas da variância (Wald $X_{(2)}^{2}=10.59, p=0.005$ ). A história de consumo de drogas é o preditor mais significativo $(z=3.11, p=0.002)$, revelando que os reclusos com história de consumo de drogas tendem a incorrer num maior número de processos disciplinares na prisão. Para além disso, verificou-se que os reclusos com níveis elevados de agressividade também se associam a um maior número de infrações $(z=1.98, p=$ 0.048), embora de forma menos significativa. Seguidamente calculamos o número previsto de processos disciplinares de acordo com os preditores do nosso modelo de regressão, que pode ser visualizado através do Gráfico 1.

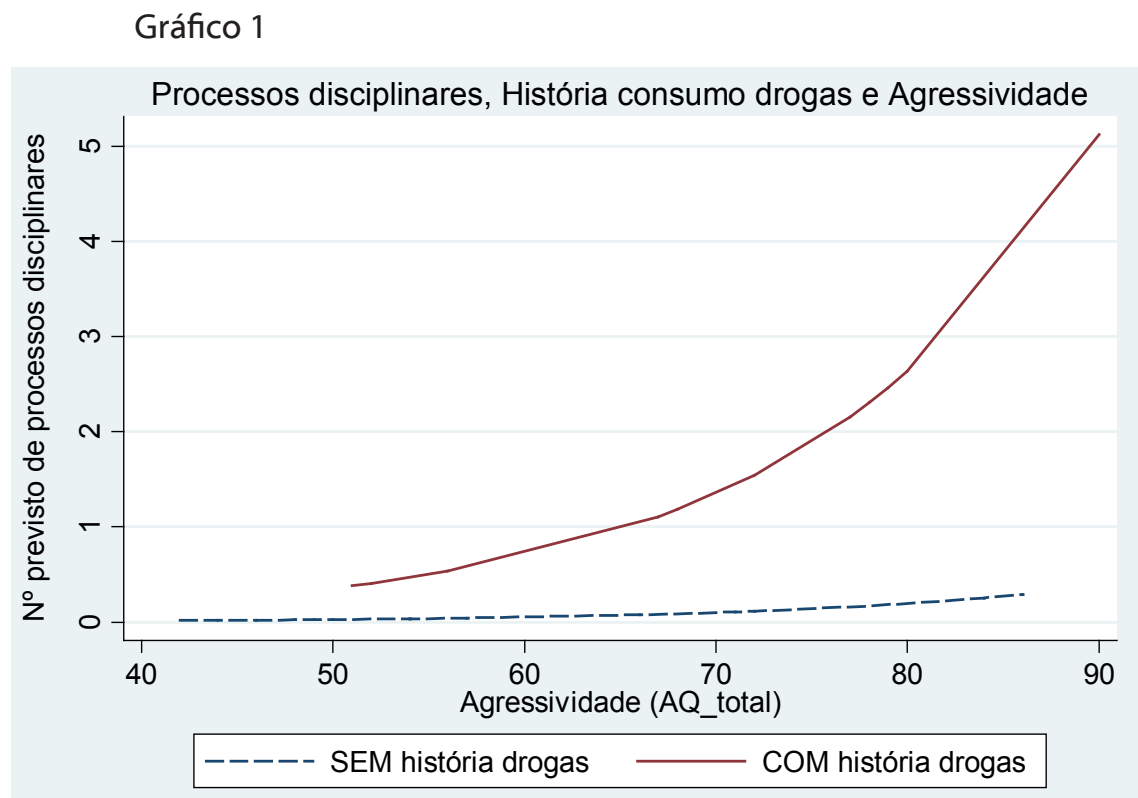

Constata-se que, para reclusos "sem história de consumo de drogas", os resultados no AQ influenciam pouco o número previsto de processos disciplinares. Ou seja, a curva dos processos disciplinares pouco aumenta com o aumento dos resultados neste teste. Especificamente, espera-se que um recluso "sem história de consumo de drogas" e com pontuação 
de 50 pontos no AQ incorra em 0.026 processos disciplinares num período de cinco meses; se o recluso pontuar 60 espera-se que incorra em 0.051 processos; se pontuar 70 espera-se que incorra em 0.1 ; se pontuar 80 espera-se que incorra em 0.194 ; e se pontuar 90 espera-se que incorra em 0.377 processos disciplinares.

Ao contrário, no grupo de reclusos "com história de consumo de drogas", constata-se que o número previsto de processos disciplinares aumenta exponencialmente com o aumento da pontuação no AQ. Especificamente, espera-se que um recluso "com história de consumo de drogas" e uma pontuação de 50 neste questionário incorra em 0.358 processos disciplinares (em 5 meses); se pontuar 60 espera-se que incorra em 0.696 processos; se pontuar 70 espera-se que incorra em 1.354; se pontuar 80 espera-se que incorra em 2.634; e se pontuar 90 espera-se que incorra em 5.124 processos disciplinares.

Quanto aos acessos aos serviços clínicos, usando modelos de regressão linear, constatamos que os preditores que explicam maior variância são a hostilidade medida pelo fator 4 do $\mathrm{AQ}\left(R_{a j}^{2}=0.24, F_{(1,29)}=10.26\right.$, $p=0.003)$ e o tempo de condenação inferior a 12 meses $\left(R_{a j}^{2}=0.096, F_{(1,29)}\right.$ $=4.18, p=0.05)$, sendo ambos significativos ${ }^{1}$. Apesar da variável dependente (serviços clínicos) cumprir os pressupostos das regressões lineares, seguimos a mesma metodologia anteriormente descrita, comparando diferentes modelos, os quais apresentamos na Tabela 3.

Tabela 3

Modelos de regressão para as variáveis Acessos aos serviços clínicos, Hostilidade e Tempo de pena por classes

\begin{tabular}{cccc}
\hline LL & gl & BIC & Modelo \\
\hline-83.31 & 3 & 176.92 & Ordinary Least Squares \\
-78.81 & 3 & 167.93 & Poisson \\
-71.24 & 4 & 156.21 & Negative Binomial \\
\hline
\end{tabular}

LL:Log-likelihood

gl: graus de liberdade

BIC: Bayesian Information Criterion

1 Quanto ao tempo de condenação, temos de realçar que este preditor foi sujeito a imputações múltiplas. Como 10 dos reclusos estão em prisão preventiva, não têm tempo de condenação atribuído. Por defeito, o software estatístico elimina os casos em falta numa determinada variável da análise de todas as restantes variáveis inseridas no modelo. Visto que a nossa amostra é reduzida, esta questão seria problemática, pois ficaríamos apenas com 21 observações. Geramos por isso 10 imputações múltiplas para completar a amostra. 
À semelhança dos achados no indicador anterior (processos disciplinares), o modelo mais adequado para analisar estas variáveis é o Negative Binomial, embora as diferenças não sejam tão acentuadas. Este modelo, analisando a hostilidade (AQ - Hostilidade) e o tempo de pena por classes enquanto preditores do número de acessos aos serviços clínicos, apresenta um Pseudo $R^{2}$ de 0.08 e é estatisticamente significativo, mesmo após calcularmos estimativas robustas da variância $\left(\right.$ Wald $X_{(4)}=30.71, p<$ $0.001)$. A hostilidade é o preditor mais significativo $(z=4.37, p=<0.001)$, o que indica que os reclusos com pontuações mais elevadas nesta escala tendem a recorrer mais vezes aos serviços clínicos. O tempo de condenação inferior a 12 meses também é significativo $(z=2.43, p=0.015)$, demonstrando que os reclusos com penas mais curtas também recorrem mais aos cuidados médicos. O gráfico 2 apresenta o número previsto de acessos aos serviços clínicos de acordo com os preditores do modelo.

Gráfico 2

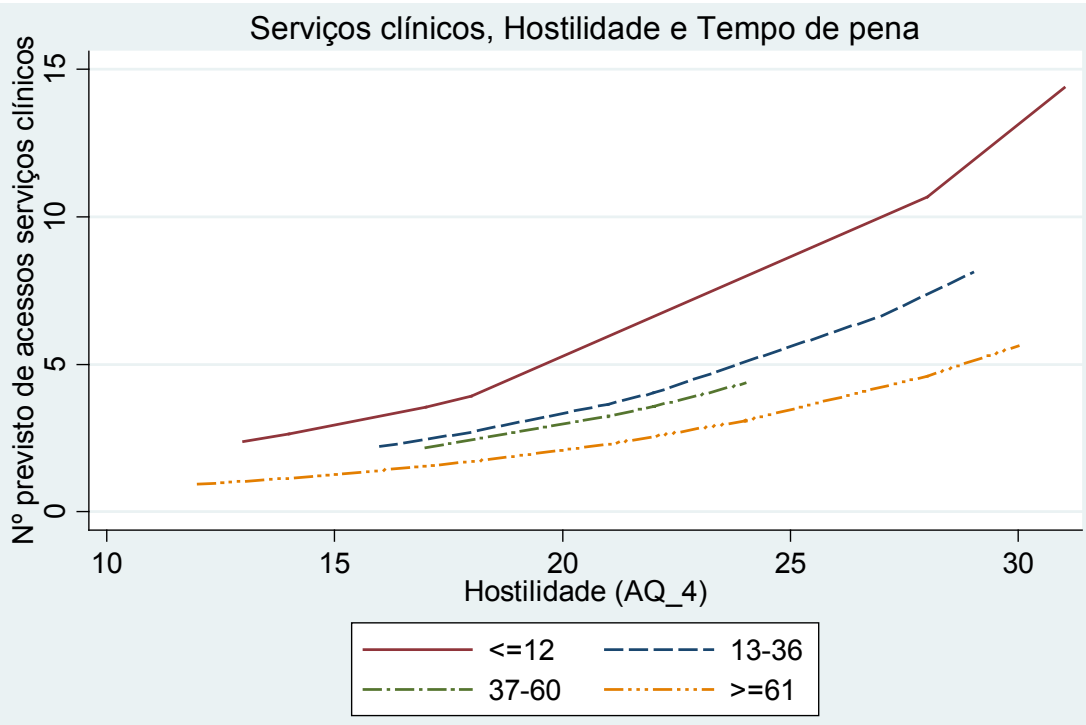

Constata-se que as curvas relativas ao número previsto de acessos aos serviços clínicos aumentam com o aumento da pontuação na escala de hostilidade. No entanto, as diferenças mais acentuadas verificam-se ao nível dos reclusos com menos de 12 meses de condenação, sendo este grupo o único estatisticamente significativo. Os outros funcionam apenas como variáveis de controle. Assim, mantendo as outras variáveis constantes, espera-se que um recluso com uma pena de prisão inferior a 12 meses e com 15 pontos na escala de hostilidade recorra 2.914 vezes aos serviços clínicos (em 5 meses); se o recluso pontuar 20 espera-se que 
recorra 4.799 vezes; se pontuar 25 espera-se que recorra 7.904 vezes; e se pontuar 30 espera-se que recorra 13.019 vezes aos serviços clínicos.

Atendendo ao segundo objetivo do estudo, procuramos identificar variáveis sociodemográficas e jurídico-penais preditoras da agressividade e do estilo de vida criminal. Para tal, utilizamos regressões lineares (visto se tratarem de variáveis dependentes intervalares e cumprirem os pressupostos destas análises) seguidas de análises robustas da variância. Deste modo, verificamos que a idade prediz marginalmente a agressividade $\left(R_{a j}^{2}=0.06, F_{(1,29)}=3.70, \mathrm{p}=0.064\right)$, ou seja, os reclusos mais jovens tendem a ser mais agressivos na prisão. Verificamos ainda que a situação penal e os antecedentes penitenciários predizem marginalmente a agressividade, demonstrando que os reclusos condenados tendem a ser mais agressivos $\left(R_{a j}^{2}=0.08, F_{(1,29)}=3.37, p=0.071\right)$, também o sendo os reclusos reincidentes $\left(R_{a j}^{2}=0.08, F_{(1,29)}=3.56, p=0.077\right)$. Verificamos ainda que 0 número de visitas dos reclusos prediz significativamente a agressividade $\left(R_{a j}^{2}=0.14, F_{(1,29)}=9.29, p=0.005\right)$, isto é, reclusos com menor número de visitas tendem a apresentar valores mais elevados de agressividade.

Após inserirmos estas variáveis num modelo de regressão, excluímos os preditores "situação penal" e "antecedentes criminais" por estarem associados entre si e perderem significância. Assim, criamos um modelo de regressão linear múltipla em que o número de visitas e a idade são os preditores da agressividade, procedendo de seguida à análise robusta da sua variância. Verificamos que este modelo prediz cerca de $22 \%$ da variância nos valores na agressividade, sendo significativo $\left(R_{a j}^{2}=0.22\right.$, $\left.F_{(2,28)}=8.28, p=0.002\right)$. O número de visitas $(t=-3.11, p=0.004)$ e a idade $(t$ $=-2.31, p=0.029$ ) são ambos preditores significativos no modelo, ou seja, reclusos com menos visitas e uma idade mais jovem tendem a ser mais agressivos na prisão. $O$ número de visitas que os reclusos recebem também prediz o estilo de vida criminal $\left(R_{a j}^{2}=0.29, F_{(1,29)}=20.82, p<0.001\right)$, ou seja, os reclusos com menos visitas tendem a apresentar resultados mais elevados no LCSF-R.

Quanto ao terceiro objetivo do estudo, verificamos que apenas o $\mathrm{AQ}$ se associa aos indicadores da adaptação à prisão aqui estudados: o total para os processos disciplinares e a escala de hostilidade para os acessos aos serviços clínicos. O mesmo não se pode dizer do LCSF-R, que não prediz nenhum dos indicadores da adaptação aqui estudados.

Debruçamo-nos em seguida sobre os resultados dos reclusos ao nível da sua adaptação à prisão para os comparar com a tipologia de Gonçalves $(1993,2008)$, em consonância com o quarto objetivo deste estudo. Na Tabela 4 apresenta-se a grelha de avaliação da adaptação à prisão que inclui a distribuição da pontuação dos reclusos pelos dois indicadores da adaptação, bem como as pontuações totais. 
Tabela 4

Grelha de Avaliação da Adaptação à Prisão

\begin{tabular}{|c|c|c|c|c|}
\hline \multicolumn{5}{|l|}{ Processos disciplinares } \\
\hline$N^{\circ}$ de processos disciplinares & 0 & $1-3$ & $4-6$ & $>6$ \\
\hline Pontuação & 1 & 0 & -1 & -2 \\
\hline $\mathrm{n}$ & 24 & 4 & 2 & 1 \\
\hline \multicolumn{5}{|l|}{ Serviços clínicos } \\
\hline$N^{\circ}$ acessos aos serviços clínicos & 0 & $1-8$ & $9-16$ & $>16$ \\
\hline Pontuação & 1 & 0 & -1 & -2 \\
\hline $\mathrm{n}$ & 5 & 23 & 1 & 2 \\
\hline ADAPTAÇÃO À PRISÃO (total) & \multicolumn{2}{|c|}{ Adaptados } & \multicolumn{2}{|c|}{ Não adaptados } \\
\hline Pontuação & \multicolumn{2}{|c|}{$0-2$} & \multicolumn{2}{|c|}{$<0$} \\
\hline $\mathrm{n}$ & \multicolumn{2}{|c|}{26} & \multicolumn{2}{|c|}{5} \\
\hline
\end{tabular}

Relativamente às pontuações totais, constatamos que 26 reclusos se classificam entre 0 e 2 (83.9\%), sendo, assim, considerados adaptados, enquanto 5 obtêm pontuações negativas (16.1\%), sendo por isso considerados não adaptados à prisão. A Tabela 5 apresenta a pontuação dos reclusos não adaptados nos dois indicadores da adaptação.

Tabela 5

Pontuação dos reclusos não adaptados na Grelha de Avaliação da Adaptação à Prisão

\begin{tabular}{cccc}
\hline Noparticipante & $\begin{array}{c}\text { Processos disciplinares } \\
\text { (Mal-adaptados) }\end{array}$ & $\begin{array}{c}\text { Serviços clínicos } \\
\text { (Inadaptados) }\end{array}$ & $\begin{array}{c}\text { Adaptação à prisão } \\
\text { (total) }\end{array}$ \\
\hline 2 & 0 & -1 & -1 \\
7 & -1 & 0 & -1 \\
14 & -1 & -2 & -3 \\
19 & -2 & 0 & -2 \\
29 & 1 & -2 & -1 \\
\hline
\end{tabular}


Verifica-se que 2 reclusos pontuam negativo ao nível dos processos disciplinares (participante 7 e 19) sendo assim considerados "mal-adaptados"; outros 2 pontuam negativo nos acessos aos serviços clínicos (participante 2 e 29) e são assim considerados "inadaptados"; por fim, 1 recluso pontua negativo tanto nos processos disciplinares como nos acessos aos serviços clínicos (participante 14) pelo que poderá ser considerado um recluso do tipo "borderline".

Salvaguardando o teor meramente descritivo desta comparação, podemos constatar algumas semelhanças entre a caracterização dos reclusos considerados como não adaptados à prisão nesta amostra e a tipologia de adaptação à prisão de Gonçalves $(1993,2008)$. Assim, notamos que os reclusos considerados "inadaptados" neste estudo apresentam resultados especialmente elevados ao nível da hostilidade. Esta dimensão denota uma componente cognitiva associada a pensamentos negativos, o que reflete o stress prisional e as patologias de adaptação descritas na tipologia. Para além disso, e apesar de um dos dois reclusos ter 36 anos, o outro tem apenas 23 , idade próxima da média de 24 anos exposta na tipologia, que associa esta categoria a reclusos mais jovens.

Relativamente aos reclusos "mal-adaptados", estes apresentam resultados elevados em todas as dimensões da agressividade e associam-se à violação das regras institucionais e aos comportamentos violentos descritos na tipologia. Para além disso, estes reclusos são reincidentes e têm idades próximas da média de 29 anos ( 28 e 32 anos, respectivamente). Verifica-se ainda que ambos estão condenados por crimes contra o património e apresentam história de consumo de álcool e drogas.

Quanto ao recluso tipificado como "borderline", este é o menos adaptado à prisão e, como esperado, possui características dos dois tipos anteriores. Assim, apresenta resultados elevados na hostilidade, à semeIhança dos "inadaptados" e, além disso, está detido por crimes contra o património e apresenta história de consumo de álcool e drogas, à semeIhança dos reclusos "mal-adaptados".

\section{Discussão}

Considerando o primeiro objetivo, constatamos que a história de consumo de drogas e a agressividade demonstram ser os preditores mais significativos das infrações na prisão. Vários estudos têm consistentemente reportado a ligação entre a droga e problemas disciplinares, relacionando-a nomeadamente com agressões contra reclusos e funcionários e esquemas de vitimização prisional (e.g. Jiang \& Fisher-Giorlando, 2005; Snacken, 2005).Também tem sido reportada a sua associação a um estilo de vida antissocial e ao crime contra o património 
(e.g. Torres \& Gomes, 2002; Ventura, 1997; Walters, 1998). Relativamente à agressividade na prisão, esta tem sido explicada através de fatores intrínsecos aos reclusos e/ou fatores associados ao meio prisional (e.g. Butler \& Kariminia, 2006; Dhami et al., 2007). Ao nível dos fatores pessoais, os nossos resultados corroboram os de estudos anteriores (Lahm, 2008; Rodríguez-Fornells, Lopez-Capdevila, \& Andres-Pueyo, 2002) em que se comprovou a associação entre a agressividade medida pelo $A Q$ e os comportamentos disruptivos na prisão. Ao nível institucional, sabe-se que a agressividade varia significativamente entre diferentes prisões, sendo mais frequente em estabelecimentos com elevadas taxas de superlotação e uma larga porcentagem de jovens adultos (Lahm, 2008).

Verificamos ainda que a hostilidade (medida pelo $A Q$ ) e o tempo de pena inferior a 12 meses predizem o número de acessos aos serviços clínicos. Noutras investigações, níveis elevados de hostilidade surgiram associados à vulnerabilidade perante a instabilidade emocional (Tremblay \& Ewart, 2004), o que poderá também explicar o elevado número de acessos aos serviços clínicos dos reclusos, atendendo ao impacto da reclusão e consequentes desequilíbrios emocionais que provoca. Num estudo realizado com estudantes, Felsten e Hill (1999) verificaram que os indivíduos com elevada hostilidade apresentam níveis mais elevados de raiva depois de uma provocação, o que também poderá indicar que estes sujeitos têm dificuldades em lidar com a frustração. Relativamente ao tempo de pena, e graças aos estudos longitudinais realizados em contexto prisional, sabe-se que é frequente ocorrerem mais distúrbios emocionais no início do cumprimento da pena, os quais se vão dissipando com o habituar ao sistema prisional (Carreira \& Gonçalves, 2010; Zamble, 1992; Zamble \& Porporino, 1988, 1990).

Relativamente ao segundo objetivo, verificamos que o número reduzido de visitas e as idades mais jovens são preditoras da agressividade na prisão. Quanto à idade, estes resultados corroboram os achados na literatura que têm identificado sistematicamente esta variável como um dos maiores preditores da violência (e.g. Jiang \& Fisher-Giorlando, 2002; Kuanlig, Sorensen, \& Cunningham, 2008; Lahm, 2008). Os reclusos mais jovens tendem a ser mais voláteis e contrários às normas, sendo mais propensos ao envolvimento em atos violentos (e.g. Butler \& Kariminia, 2006). Quanto ao número de visitas, não conhecemos nenhum estudo que apresente resultados entre esta variável e a agressividade. No entanto, existem dados que sugerem que as visitas recebidas pelos reclusos ajudam a reduzir e retardar a reincidência no crime (Bales \& Mears, 2008). Para além disso, os reclusos relatam que as visitas os ajudam a criar amizades e a lidar com a vida na prisão (Duncan \& Balbar, 2008).

Verificamos ainda que os reclusos com resultados mais elevados ao nível do estilo de vida criminal tendem a associar-se a um número reduzido de visitas. Este resultado pode indiciar ruptura dos laços com a rede 
familiar e social de origem, sendo este um elemento que ajuda igualmente a perpetuar um estilo de vida antissocial e a reincidência no crime.

Atendendo ao terceiro objetivo, verificamos que o LCSF-R não prediz a adaptação à prisão melhor do que o acaso, como já reportara o seu autor (Walters, 2005, 2007). O estilo de vida criminal é um construto que se apoia num conjunto de atitudes e comportamentos que o sujeito vai perpetuando ao longo da sua existência. Salienta-se que na nossa amostra apenas dois sujeitos atingiram o limiar proposto pelo autor (10 p.) para serem identificados como possuidores de um estilo de vida criminal. De fato, cerca de metade da amostra não teve condenações anteriores em pena de prisão, o que contribui para resultados mais baixos neste instrumento e pode, também, influenciar o modo de cumprimento da pena. Pelo contrário, o AQ parece estar associado tanto aos processos disciplinares como aos acessos aos serviços clínicos, o que atesta a sua utilidade no contexto prisional. Apesar de se tratar de uma amostra pequena, estes dados parecem corroborar a utilidade dos instrumentos de autorrelato quando aplicados a amostras forenses.

No que toca à caracterização de diferentes tipologias da adaptação à prisão, constatou-se que a esmagadora maioria dos reclusos desta amostra tende a ser adaptada à vida dentro do estabelecimento prisional, na sequência aliás de estudos anteriores (Gonçalves, 2008). Este fato poderá explicar-se tanto pelas características da amostra como pelo próprio estabelecimento prisional, destinado sobretudo a reclusos preventivos ou com penas de prisão relativamente curtas. Para além disso, é um estabelecimento muito pequeno e bastante vigiado, reduzindo muitos dos tradicionais problemas das prisões de maior dimensão (e.g. drogas, contrabando, exploração econômica e física, motins, fugas, etc.). Relativamente aos reclusos não adaptados desta amostra, estes apresentam semelhanças com a tipologia de Gonçalves $(1993,2008)$ e atestam a utilidade que as tipologias podem ter na compreensão de diferentes perfis de adaptação à prisão.

\section{Conclusão}

Antes de expor as conclusões deste estudo, é importante referir as suas limitações. A amostra é reduzida e todos os reclusos pertencem à mesma prisão, o que causa entraves quanto à representatividade dos fenômenos estudados e generalização dos resultados. A exiguidade da amostra acarreta também limitações no nível das análises estatísticas, devido a não se cumprirem determinados pressupostos. Quanto ao $A Q$, por ser um questionário, não está imune ao fenômeno da desejabilidade social, o que poderá estar associado aos baixos resultados obtidos pela 
amostra neste instrumento. Além disso, o fato de a estrutura fatorial da versão portuguesa do LCSF-R (Vieira et al., 2008) só parcialmente replicar a do instrumento original impõe também que sejam acauteladas generalizações abusivas. Podemos ainda referir que o período de observação é relativamente curto para avaliar a adaptação à prisão, pois esta é um processo longitudinal e suscetível a alterações ao longo do tempo. Por fim, o preditor "tempo de condenação" foi sujeito a dez imputações múltiplas, que são estimativas e não resultados reais.

Ainda assim, dada a natureza exploratória do presente estudo, consideramos que a sua metodologia se adequa aos objetivos de investigação traçados e, nesse sentido, tentaremos providenciar algumas recomendações para o tratamento e gestão penitenciária dos reclusos. Em primeiro lugar, o AQ pode ser relevante na avaliação dos reclusos, pois está associado ao número de processos disciplinares e ao número de acessos aos serviços clínicos. Assim, pode ser utilizado na classificação dos reclusos em diferentes níveis de segurança, pois vimos que quando as pontuações elevadas se associam à história de consumo de drogas, a previsão dos processos disciplinares tende a subir exponencialmente. Para além disso, verificamos que a escala de hostilidade deste instrumento prevê o número de acessos aos serviços clínicos, sobretudo no caso de reclusos com penas de prisão inferiores a 12 meses. Neste sentido, poderia também ajudar a sinalizar reclusos com necessidade de acompanhamento clínico mais imediata.

Olhando para a gestão dos reclusos, parece-nos claro que diferentes percursos de adaptação à prisão requerem cuidados penitenciários diferenciados. De fato, os reclusos "mal-adaptados" necessitam de maior vigilância e intervenção psicoeducativa, nomeadamente programas de autocontrole, treino de competências sociais e estratégias de resolução dos problemas. Apesar de alguns esforços na criação e implementação de programas prisionais, estes são em número ainda bastante reduzidos em Portugal e não se aplicam em todos os estabelecimentos prisionais. Por seu lado, os reclusos "inadaptados" carecem sobretudo de cuidados médicos e psicológicos, como serviços de enfermagem e consultas de psicologia e psiquiatria. No entanto, em estabelecimentos prisionais como o deste estudo, que não possui instalações para prestação de cuidados especializados e constantes, é difícil providenciar o acompanhamento necessário aos reclusos mais sintomáticos. Quanto aos reclusos "borderline", estes necessitam tanto de intervenção psicoeducativa como de cuidados médicos e psicológicos, sendo estes os reclusos sobre os quais deve incidir maior foco de atenção ao nível dos serviços clínicos, educativos e de vigilância, visto serem os mais inconstantes e menos adaptados à prisão.

Tal como noutros contextos carcerários, verificamos na nossa amostra que a história de consumo de drogas se associa fortemente às infrações institucionais, pelo que os programas de tratamento da toxi- 
codependência desenvolvidos em meio prisional são essenciais para o sucesso na adaptação à prisão destes reclusos, devendo ser implementados preferencialmente logo no início da detenção. Para além disso, atendendo à associação significativa entre o número reduzido de visitas e os níveis elevados de agressividade e estilo de vida criminal, seria de todo o interesse promover mais programas de visitantes nos diversos estabelecimentos prisionais, pois são de baixo custo e, de acordo com a literatura, parecem ajudar os reclusos a lidar com a prisão e manterem-se afastados da criminalidade.

Concluímos salientando que a avaliação do risco deve nortear a apreciação dos reclusos, tanto em termos de adaptação à prisão como no regresso à liberdade. Apesar das potencialidades do $A Q$, instrumentos mais estruturados como o Level of Service Inventory - Revised (Andrews \& Bonta, 1995) e o Historical Clinical Risk - 20 (Webster et al., 1997) poderão ser particularmente úteis (cf. Farrington, Jolliffe, \& Johnstone, 2008; Neves, Gonçalves, \& Palma-Oliveira, 2011).

\title{
Aggressiveness, criminal lifestyle and prison adjustment
}

\begin{abstract}
The study of inmates' adjustment to prison and prison classification may help to prevent risky situations and promote a more effective institutional treatment. This correlational study explores the relation between aggressiveness, criminal lifestyle, and adjustment to prison, attending also to socio-demographic and penal variables. Aggressiveness was measured through the Aggression Questionnaire and the Lifestyle Criminality Screening Form-Revised assessed the criminal lifestyle. Prison adjustment includes two indicators: infraction reports and accesses to the clinical services. A sample of thirty-one Portuguese male prisoners from a local prison was assessed during an observation period of five months. Results show that aggressiveness, drug history, and shorter sentences predict a poor adjustment. The scores on Aggression Questionnaire are associated with both infractions reports and accesses to the clinical services.
\end{abstract}

Keywords: Adjustment. Aggressiveness. Lifestyle. Prison. 


\section{Agressivité, style de vie criminel et adaptation à la prison}

Résumé: Les études sur l'adaptation à la prison et la classification des détenus peuvent aider à prévenir des situations de risque et promouvoir un traitement pénitentiaire plus efficient. Cette recherche corrélationnelle explore la relation entre l'agressivité, le style de vie criminel et l'adaptation à la prison, incluant des variables socio-démographiques et juridico-pénales. L'agressivité est mesurée par l'Agression Questionnaire et le style de vie criminelle par le Lifestyle Criminality Screening Form. L'adaptation à la prison comprend deux indicateurs: les procédures disciplinaires et les accès aux services cliniques. L'échantillon se compose de trente et un participants du sexe masculin et de nationalité portugaise, incarcéré dans une prison régionale et observé durant une période de cinq mois. Les résultats indiquent que l'agressivité, I'histoire d'usage de drogues et les peines plus courtes prédisent une mauvaise adaptation à la prison et que l'Agression Questionnaire est associé aux procédures disciplinaires et aux accès aux services cliniques.

Mots-clés: Adaptation. Agressivité. Style de vie. Prison.

\section{Agresividad, estilo de vida criminal y adaptación a la prisión}

Resumen: El estudio de la adaptación a la prisión y la clasificación de los reclusos pueden ayudar a prevenir situaciones de riesgo y promover un tratamiento penitenciario más adecuado. Este estudio correlacional explora la relación entre la agresividad, el estilo de vida criminal y la adaptación a la prisión, incluyendo datos socio-demográficos y jurídico-penales. La agresividad es medida por el Aggression Questionnaire y lo estilo de vida criminal por el Lifestyle Criminality Screening Form. La adaptación a la prisión incluye dos indicadores: los procedimientos disciplinarios y los accesos a los servicios clínicos. La muestra consta de treinta y uno participantes del sexo masculino y nacionalidad portuguesa, detenidos en una prisión regional. El periodo de observación fue de cinco meses. Los resultados indican que la agresividad, la historia del consumo de drogas y condenas más cortas predicen peor adaptación a la cárcel. Además se verifica que los scores Aggression Questionnaire se asocian con los procedimientos disciplinarios y el acceso a los servicios clínicos.

Palabras clave: Adaptación. Agresividad. Estilo de vida. Prisión. 


\section{Referências}

American Psychiatric Association. (2002). DSM-IV-TR: Manual de diagnóstico e estatística das perturbações mentais. Lisboa: Climepsi.

Andrews, D. A., \& Bonta, J. (1995). LSI-R: The Level of Service Inventory-Revised. Toronto: Multi-Health Systems.

Bales, W. D., \& Mears D. P. (2008). Inmate social ties and the transition to society: Does visitation reduce recidivism? Journal of Research in Crime and Delinquency, 45(3), 287-321. doi: 10.1177/0022427808317574

Buss, A. H., \& Perry, M. (1992). The Aggression Questionnaire. Journal of Personality and Social Psychology, 3, 452-459. doi:10.1037/0022-3514.63.3.452

Butler, T., \& Kariminia, A. (2006). Prison violence: Perspectives and epidemiology. New South Wales Public Health Bulletin, 17(1-2), 17-20. doi:10.1071/NB06005

Carbonell, L. J., Megargee, E. I., \& Moorhead, K. M. (1984). Predicting prison adjustment with structured personality inventories. Journal of Consulting and Clinical Psychology, 2, 280-294.

Carreira, L., \& Gonçalves, R. A. (2010). Psicopatologia e adaptação à prisão. Saúde Mental, 12(4), 14-30.

Clemmer, D. (1940). The prison community. New York: Holt, Rinehart \& Winston.

Coleman, R. (2008). Carceral society. In Y. Jewkes \& J. Bennett (Eds.), Dictionary of prisons and punishment (p. 32). Cullompton: Willan.

Dhami, M. K., Ayton, P., \& Loewenstein, G. (2007). Adaptation to imprisonment: Indigenous or imported? Criminal Justice and Behavior, 34(8), 1085-1100. doi: $10.1177 / 0093854807302002$

Duncan, H. E., \& Balbar, S. (2008). Evaluation of a visitation program at a Canadian penitentiary. The Prison Journal, 88(2), 300-327. doi:10.1177/0032885508319210

Farrington, D. P., Jolliffe, D., \& Johnstone, L. (2008). Assessing violence risk: A framework for practice. Final report to the U.S. Department of Justice.

Felsten, G., \& Hill, V. (1999). Aggression Questionnaire hostility scale predicts anger in response to mistreatment. Behaviour Research and Therapy 1, 87-97. 
Gallardo-Pujol, D., Kramp, U., García-Forero, C., Pérez-Ramírez, M., \& Andrés-Pueyo, A. (2006). Assessing aggressiveness quickly and efficiently: the Spanish adaptation of Aggression Questionnaire-Refined version. European Psychiatry, 21(7), 487494. doi: 10.1016/j.eurpsy.2006.02.002

García-León, A., Reyes, G. A., Vila, J., Pérez, N., Robles, H., \& Ramos, M. M. (2002). The Aggression Questionnaire: A validation study in student samples. The Spanish Journal of Psychology, 5(1), 45-53.

Garrido, G. V. (1993). Técnicas de tratamiento para delinquentes. Madrid: Centro de Estudios Ramon Areces.

Goffman, E. (1961). Asylums. New York: Anchor Books.

Gonçalves, R. A., \& Vieira, S. (2005). A avaliação do estilo de vida criminal em ofensores sexuais. Psicologia: Teoria, Investigação e Prática, 10(1), 81-92.

Gonçalves, R. A. (1993). A adaptação à prisão: um processo vivido e observado. Lisboa: Direcção-Geral dos Serviços Prisionais.

Gonçalves, R. A. (2008). Delinquência, crime e adaptação à prisão (3a ed.). Coimbra: Quarteto.

Gonçalves, R. A., \& Lopes, M. (2007). Mulheres na prisão e reprodução da criminalidade. In Actas do Seminário "Educar o Outro" - As questões de género, dos direitos humanos da educação nas prisões portuguesas (pp. 55-77). Coimbra: Humana Global. Recuperado de www.humanaglobal.org

Haney, C. (2003). The psychological impact of incarceration: Implications for postprison adjustment. In J. Travis \& M. M. Waul (Eds.), From prisoners once removed: The impact of incarceration and reentry on children, families, and communities (pp. 33-66). Washington, DC: Urban Institute.

Harding, T., \& Zimmerman, E. (1989). Psychiatric symptoms, cognitive stress, and vulnerability factors: A study in a remand prison. British Journal of Psychiatry, 155, 36-43.

Hochstetler, A., \& DeLisi, M. (2005). Importation, deprivation, and varieties of serving time: An integrated-lifestyle-exposure model of prison offending. Journal of Criminal Justice 33, 257-266. doi: 10.1016/j.jcrimjus.2005.02.005

Ireland, J. L., Brown, S. L., \& Ballarini, S. (2006). Maladaptive personality traits, coping styles and psychological distress: A study of adult male prisoners. Personality and Individual Differences, 41(3), 561-573. 
Irwin, J., \& Cressey, D. (1962). Thieves, convicts, and the inmate culture. Social Problems, 10, 142-155.

Jewkes, Y. (2008). Prisonization. In Y. Jewkes \& J. Bennett (Eds.), Dictionary of prisons and punishment (pp. 210-211). Cullompton: Willan.

Jiang, S. (2005). Impact of drug use on inmate misconduct: A multilevel analysis. Journal of Criminal Justice, 33, 153-163. doi:10.1016/j.jcrimjus.2004.12.007

Jiang, S., \& Fisher-Giorlando, M. (2002). Inmate misconduct: A test of the deprivation, importation, and situational models. The Prison Journal, 82(3), 335-358. doi: $10.1177 / 003288550208200303$

Kuanliang, A., Sorensen, J. R., \& Cunningham, M. D. (2008). Juvenile inmates in an adult prison system: Rates of disciplinary misconduct and violence. Criminal Justice and Behavior, 35(9), 1186-1201. doi: 10.1177/0093854808322744

Lahm, K. F. (2008). Inmate-on-inmate assault: A multilevel examination of prison violence. Criminal Justice and Behavior, 35(1), 120-137. doi: $10.1177 / 0093854807308730$

MacKenzie, D. (1989). Prison classification: The management and psychological perspectives. In L. Goodstein \& D. L. MacKenzie (Eds.), The american prison: Issues in research and policy (pp. 163-189). New York: Plenum.

Motiuk, L. L. (1997). Classification for correctional programming: The Offender Intake Assessment (OIA) process. Forum on Corrections Research, 9(1), 13-22.

Neves, A. C., Gonçalves, R. A., \& Palma-Oliveira, J. M. (2011). Assessing risk for violent and general recidivism: A study of the HCR-20 and the PCL-R with a non-clinical sample of portuguese offenders. International Journal of Forensic Mental Health, 10: 2, 137-149. doi: 10.1080/14999013.2011.577290

Rodríguez-Fornells, A., Lopez-Capdevila, J. M., \& Andres-Pueyo, A. (2002). Personalidad y comportamiento penitenciario. Psicothema, 14, 90-100.

Schrag, C. (1961). A preliminary criminal typology. Pacific Sociological Review, 1, 11-16.

Simões, A. (1993). São os homens mais agressivos que as mulheres? Revista Portuguesa de Pedagogia, 27(3), 387-404.

Snacken, S. (2005). Forms of violence and regimes in prison: Report of research in Belgian prisons. In A. Liebling \& S. Maruna (Eds.), The effects of imprisonment (pp. 306-339). Devon: Willan. 
Sykes, G. (1958). The society of captives. Princeton, NJ: Princeton University Press.

Torres, A. C., \& Gomes, M. C. (2002). Drogas e prisões: "Relações perigosas". Temas Penitenciários, 2, 8-24.

Tremblay, P., \& Ewart, L. (2005). The Buss and Perry Aggression Questionnaire and its relations to values, the Big Five, provoking hypothetical situations, alcohol consumption patterns, and alcohol expectancies. Personality and Individual Differences, 2, 337-346. doi 10.1016/j.paid.2004.04.012

Ventura, J. P. (1997). Toxicodependência, motivação, comportamento delituoso e responsabilidade criminal: Alguns nexos de comprovada causalidade. Revista Portuguesa de Ciência Criminal, 7, 461-482.

Vieira. S., Lopes, M., \& Gonçalves, R. A. (2008). La evaluación del estilo de vida criminal en una muestra de presos Portugueses. In F. J. Rodriguez, C. Bringas, F. Fariña, R. Arce \& A. Bernardo (Eds.), Psicología jurídica: entorno judicial y delincuencia (pp. 359-364). Oviedo: Ediciones de la Universidad de Oviedo.

Walmsley, R. (2009). World prison population list (8th ed.). London: International Centre for Prison Studies.

Walters, G. D. (1995). Factor structure of the Lifestyle Criminality Screening Form. International Journal of Offender Therapy and Comparative Criminology, 39, 99108.

Walters, G. D. (1998). Changing lives of crime and drugs intervening with substanceabusing offenders. Chichester: Wiley.

Walters, G. D. (2005). Predicting institutional adjustment with the Lifestyle Criminality Screening Form and Psychological Inventory of Criminal Thinking Styles. International Journal of Forensic Mental Health, 4(1), 63-70.

Walters, G. D. (2007). Predicting institutional adjustment with the Lifestyle Criminality Screening Form and the Antisocial Features and Aggression scales of the PAI. Journal of Personality Assessment, 88, 99-105. doi:10.1080/00223890709336840

Webster, C. D., Douglas, K. S., Eaves, D., \& Hart, S. D. (1997). HCR-20: Assessing risk for violence (version 2). Vancouver, Canada: Simon Fraser University.

Wright, K. (1985). Improving correctional classification through a study of the placement of inmates in environmental settings. National Institute of Justice Final Report No. 83-IJ-CX-0011). Washington, DC: U.S. Department of Justice. 
Zamble, E. (1992). Behavior and adaptation in long-term prison inmates: Descriptive longitudinal results. Criminal Justice and Behavior, 19, 409-425. doi: 10.1177/0093854892019004005

Zamble, E., \& Porporino, F. J. (1988). Coping, behavior, and adaption in prison inmates. New York: Springer.

Zamble, E., \& Porporino, F. J. (1990). Coping, imprisonment, and rehabilitation: Some data and their implications. Criminal Justice and Behavior, 17(1), 53-70. doi: $10.1177 / 0093854890017001005$ 
Leonel Cunha Gonçalves, doutorando em Psicologia da Justiça da Escola de Psicologia da Universidade do Minho. Bolsista da Fundação para a Ciência e a Tecnologia, Braga, Portugal. Endereço para correspondência: Universidade do Minho Campus de Gualtar, CEP 4710-057,Braga, Portugal.Endereço eletrônico: leonel.cg@gmail.com

Rui Abrunhosa Gonçalves, professor Associado com Agregação da Escola de Psicologia da Universidade do Minho, Braga, Portugal. Endereço para correspomdência: Escola de Psicologia, Universidade do Minho Campus de Gualtar, CEP 4710-057, Braga, Portugal.Endereço eletrônico: rabrunhosa@psi.uminho.pt 